\title{
An enantiomer-based virtual screening approach: Discovery of chiral organophosphates as acetyl cholinesterase inhibitors
}

\author{
Aiqian Zhang ${ }^{\mathrm{a}}$, Yunsong $\mathrm{Mu}^{\mathrm{b}, *}$, Fengchang $\mathrm{Wu}^{\mathrm{b}}$ \\ a State Key Laboratory of Environmental Chemistry and Ecotoxicology, Research Center for Eco-Environmental Sciences, Chinese Academy of Sciences, Beijing 100085, \\ China \\ b State Key Laboratory of Environmental Criteria and Risk Assessment, Chinese Research Academy of Environmental Sciences, Beijing 100012, China
}

\section{A R T I C L E I N F O}

\section{Keywords:}

Chiral organophosphates

Enantioselectivity

In vitro testing

Molecular docking

Virtual screening

\begin{abstract}
A B S T R A C T
Chiral organophosphates (OPs) have been used widely around the world, very little is known about binding mechanisms with biological macromolecules. An in-depth understanding of the stereo selectivity of human AChE and discovering bioactive enantiomers of OPs can decrease health risks of these chiral chemicals. In the present study, a flexible molecular docking approach was conducted to investigate different binding modes of twelve phosphorus enantiomers. A pharmacophore model was then developed on basis of the bioactive conformations of these compounds. After virtual screening, twenty-four potential bioactive compounds were found, of which three compounds (Ethyl p-nitrophenyl phenylphosphonate (EPN), 1-naphthaleneacetic anhydride and $N, 4$ dimethyl- $N$-phenyl-benzenesulfonamide) were tested by use of different in vitro assays. $S$-isomer of EPN was also found to exhibit greater inhibitory activity towards human AChE than the corresponding $R$-isomer. These findings affirm that stereochemistry plays a crucial role in virtual screening, and provide a new insight into designing safer organ phosphorus pesticides on human health.
\end{abstract}

\section{Introduction}

Organophosphates (OPs) have been used to treat various neurological diseases such as Alzheimer's disease, as well as being used to prepare insecticides and fire retardant materials (Brown et al., 1989). Given that OPs are used in such a wide range of applications, questions continue to be asked regarding their biological effects, duration of action and toxic mechanisms (Liu et al., 2005). Modern OPs-based pesticides that contained one or more chiral atoms were reported to show different toxic potency to insects or human (Tenberken et al., 2010; Tsai et al., 2010; Wang et al., 2010; Zhang et al., 2014). Currently, the OPs stockpile worldwide is estimated at $200,000 \mathrm{t}$ (Singh, 2009). These concerns about health and the environment have encouraged the search for efficient tools for the environmentally benign detection of OPs (Li et al., 2014; de Castro et al., 2016). The mechanism of action of organophosphates (OPs), as well as their metabolites, has been studied using experimental and computational methods. They indicated that hydrogen bonds between $\mathrm{P}=\mathrm{O}$ and oxyanion hole was critical for AChE inhibition through covalent bond (Zhang et al., 2002; Lee and Barron, 2016). The oxyanion hole (i.e. Gly120, Gly121 and Gly122) contributed to the functional architecture and to the hydrolytic efficiency of human AChE (Ordentlich et al., 1998). Compared to $P=S$ forms, $\mathrm{P}=\mathrm{O}$ compounds are more active. The reason is that oxidation of $\mathrm{P}=\mathrm{S}$ to the corresponding $\mathrm{P}=\mathrm{O}$ results in a more electronegative phosphorus atom.

X-ray crystal structure of acetyl cholinesterase (AChE) has been solved together with several structurally diverse co-crystal structures, including tacrine (Harel et al., 1993), 1-benzyl-4-[(5,6-dimethoxy-1indanon-2-yl)methyl]piperidine (Kryger et al., 1998) and several OPs (Millard et al., 1999a, 1999b). It was a major way to acquire crucial information on the binding modes in the active site of the enzyme. The active site of AChE comprised a catalytic subsite and an anionic subsite, which binds the quaternary group of acetylcholine (Quinn, 1987). Like other serine hydrolases, the catalytic triad is located at the bottom of a deep and narrow cavity, named the "aromatic gorge". The primary site of interaction of the quaternary group of acetylcholine is with the aromatic ring of the Trp84. The $\mathrm{x}$-ray crystallographic evidence showed the presence of Trp84 and Phe330 in the "anionic" subsite of the active site and a distal residue, Trp279, near the top of the gorge, was also a part of the "peripheral" anionic site (Sussman et al., 1991). Molecular simulation approach was another way to investigate the orientation of these inhibiters to AChE (Rosenberry et al., 1999; Pilger et al., 2001; Dvir et al., 2002; Cavalli et al., 2004). This process occurred in an enantioselective manner, as exemplified by the $S$-enantiomer of an

\footnotetext{
*Correspondence to: State Key Laboratory of Environmental Criteria and Risk Assessment, Chinese Research Academy of Environmental Sciences, 8 Dayangfang, Beiyuan Road, Chaoyang District, Beijing 100012, China.

E-mail address: muyunsong323@163.com (Y. Mu).
} 
extremely poisonous gas called sarin, which phosphorylated the tyrosineosine within the active site of AChE at least 4200-fold faster than the corresponding $R$-enantiomer (Boter and Dijk, 1969). A more complicated array of interactions is involved in the phosphorylation of this enzyme to diastereoisomers of soman (Ordentlich et al., 1999). It demonstrates that AChE can discriminate between different enantiomers of OPs in terms of their toxicity towards the active site of AChE.

Enantiospecific toxicity is considered as a part of the risk assessment and regulatory decision-making processes underlying the development and application of chiral pesticides. Several quantitative structureactivity relationship (QSAR) studies have been reported pertaining to the specificity and orientation of tetrahedral alkyl phosphate in cholinesterase (Millard et al., 1999a; Ordentlich et al., 2005). Authors of these studies tried to explain the relationship between the shape of the active site of the AChE enzyme and the stereo selectivity of its binding interactions. These events were dependent on the dimensions of the acyl pocket, which governed the leaving group orientation and the productive association of the phosphonyl oxygen with the oxyanion hole (Wong et al., 2000). Comparative molecular field analysis has also used to model the binding affinities of $30 \mathrm{OPs}$, but the researchers neglected to consider the differences in the stereo chemistries of the OPs when overlaying the different conformations (Guo et al., 2006). With this in mind, the aim of the current study was to develop a new molecular modeling method for the discovery of new AChE inhibitors. Subsequent determination of the inhibitory activities of these compounds against AChE by use of an in vitro bioassay would be used to confirm the validity of this approach. Different enantiomers of chiral OPs can be used to probe the enantioselectivity of the binding pocket of AChE towards these compounds. The molecular simulation approach developed in this study could therefore be used to predict the enantiospecificity of AChE towards various chiral compounds, which can be otherwise difficult to determine experimentally.

\section{Materials and methods}

\subsection{Reagents}

Ethyl $p$-nitrophenyl phenylphosphonate (EPN, 96.5\%) was purchased from the Dr Ehrenstorfer Co. (Augsburg, Germany). Human AChE (EC 3.1.1.7) expressed in human embryonic kidney 293, acetylthiocholine iodide ( $\geq 98 \%$ ), 1-naphthaleneacetic anhydride (96\%), $N$,4-dimethyl- $N$-phenyl-benzenesulfonamide $(\geq 96 \%)$, tacrine hydrochloride ( $\geq 99 \%$ ), dimethyl sulfoxide (DMSO, $\geq 99 \%$ ), 2,2'-dinitro$5,5^{\prime}$-dithiobenzoic acid (DTNB, $\geq 97.5 \%$ ) and sodium dodecyl sulfate ( $\geq 98.5 \%$ ) were purchased from Sigma-Aldrich (St. Louis, MO, USA). Phosphate buffer solution ( $\mathrm{pH}$ 8.0) was prepared by use of solutions of $\mathrm{NaCl}, \mathrm{KCl}, \mathrm{KH}_{2} \mathrm{PO}_{4}$ and $\mathrm{Na}_{2} \mathrm{HPO}_{4}$. All of the reagents used in this study were prepared as the analytical grade.

\subsection{Theoretical calculations}

Six pairs of chiral organophosphates evaluated in this study (Hosea et al., 1995; Yen et al., 2003; Liu et al., 2006; Nillos et al., 2007) were shown in Table 1, where the $S / R$-activities had been described as the quotient of the $S$ - and $R$-activities. Three dimensional 3D structures of these OPs were constructed by use of the SYBYL X1.1 molecular modeling software package (Tripos, Princeton, NJ 08540, USA). Chemical structures were first geometrically optimized by use of the standard Tripos force field with a conjugate-gradient energy convergence criterion of $0.05 \mathrm{kcal} / \mathrm{mol}$. Atomic charges were calculated by use of the Gasteiger + Hückel charge field. The X-ray crystal structure of human AChE was obtained from the Protein Data Bank (http://www. rcsb.org/pdb), in which the protein data file entitled accession number $2 \times 8$ B (Carletti et al., 2010). Biopolymer package was used in the current study to identify the key amino acid residues involved in the binding of the co-crystallized ligand to the protein. Amino acid residues within $6.5 \AA$ around the ligand were considered during this process. Different binding modes of the chiral OPs to AChE were investigated by use of the Surflex-Dock program (Jain, 2003). Surflex-Dock's scoring function, which was based on hydrophobic, polar, repulsive, entropic and salvation terms, was trained as the total score (TS). Total score was then used to estimate the dissociation constant $K_{\mathrm{d}}$, which was expressed in units of $-\log K_{\mathrm{d}}$. In addition to the automated docking procedure, this function was recently enhanced by the incorporation of a base fragment matching algorithm. In this case, the fragments are allowed to shift from their original position during the pose optimization process.

A DISCOtech program was used to align bioactive conformations, including those of $R$-trichloronate, $S$-leptophos, $R$-fonofos, $S$-cycloheptyl thiocholine, $S$-isopropyl thiocholine, and $S$-cycloheptyl methyl Sethyl phosphonyl thioate. Bioactive conformations of these compounds generated from the Surflex-Dock program were treated as the initial configurations for establishing 50 low energy conformations. The DISCOtech program initially assigned as a series of pharmacophore features, such as hydrogen bond donor atoms, hydrogen bond acceptor atoms, charged centers, mass centers of the hydrophobic rings, and the most likely locations of the binding sites. In some cases, the chirality check option was switched on prior to performing the search process, although this was dependent on the multi-search method. In this study, the most common substructure for elements within a series of aligned conformations was superimposed by use of the categories algorithm. The 'features by class' option was defined and the number of pharmacophore features required in each class was set to more than two donor sites, as well as one acceptor atom, and one hydrophobic center. Distance tolerance between two pharmacophore features in the overlaid structures was set to $0.25 \AA$, which was increased up to $0.5 \AA$ if no model was found by use of the lower tolerance settings (step size $0.25 \AA$ ). The National Cancer Institute database was searched by use of the UNITY program, which contained two hundred thousand chemical structures at the time of this search.

\subsection{AChE competitive inhibition assay}

A colorimetric assay based on the DTNB-mediated reduction of acetylthiocholine iodide was used to measure the inhibitory activity of

Table 1

Observed $v s$. predicted $S / R$-enantiomers of organophosphates docked with acetyl cholinesterase (AChE).

\begin{tabular}{|c|c|c|c|c|c|c|}
\hline OPs & S-activity & $R$-activity & Observed $S / R$ & S-docking & $R$-docking & Predicted $S / R$ \\
\hline trichloronate & 8. $40 \pm 0.12^{\mathrm{a}}$ & $9.31 \pm 0.13^{\mathrm{a}}$ & 0.90 & 4.82 & 5.37 & 0.90 \\
\hline leptophos & $10.05^{\mathrm{a}}$ & $8.74^{\mathrm{a}}$ & 1.15 & 5.46 & 4.88 & 1.12 \\
\hline fonofos & $7.85 \pm 0.01^{\mathrm{a}}$ & 9. $04 \pm 0.10^{\mathrm{a}}$ & 0.87 & 4.13 & 4.62 & 0.89 \\
\hline cycloheptyl thiocholine & $8.28 \pm 0.07^{\mathrm{b}}$ & $5.91 \pm 0.05^{\mathrm{b}}$ & 1.40 & 7.65 & 5.78 & 1.32 \\
\hline isopropyl thiocholine & $7.20 \pm 0.03^{\mathrm{b}}$ & $5.15 \pm 0.10^{\mathrm{b}}$ & 1.40 & 3.99 & 2.92 & 1.37 \\
\hline cycloheptyl methyl S-ethyl phosphonyl thioate & $4.88 \pm 0.03^{\mathrm{b}}$ & $2.25 \pm 0.05^{\mathrm{b}}$ & 2.17 & 5.40 & 3.23 & 1.67 \\
\hline
\end{tabular}

Note:

a The logarithm of the concentration that caused 50\% mortality of the test population ( $\mu \mathrm{g} / \mathrm{L}$ ) (Yen et al., 2003; Liu et al., 2006; Nillos et al., 2007);

b The logarithm of bimolecular rate constants $\left(\mathrm{min}^{-1} \mathrm{M}^{-1}\right.$ ) determined for the inhibition of cholinesterase by $S$ - and $R$-enantiomers (Hosea et al., 1995 ). 
these compounds against AChE (Ellman et al., 1961). The reduction of acetylthiocholine iodide with DTNB resulted in the formation of thiocholine, which gave a yellow anion as an indicator of enzymatic activity. All of these experiments were conducted in triplicate, with each experiment including a positive control (tacrine) and a solvent blank control (DMSO). Forty micro liters of AChE $(0.01 \mathrm{U} / \mu \mathrm{L})$ in phosphate buffer and 100 micro liters of DTNB $(2.5 \mathrm{mmol} / \mathrm{L})$ were added to the wells of a 96-well micro plate. Twenty micro liters of tacrine in DMSO was added to each well, followed a solution of the sample extracts in DMSO $(1.10 \mathrm{~g} / \mathrm{mL}, \geq 99.9 \%)$, and the plate was then cultured in a thermo shaker (PST-60 HL plus, BIOSAN, Warren, MI 48091-1351, Latvia) at $310 \mathrm{~K}$ for $0.5 \mathrm{~h}$. Serineial dilutions of tacrine (in DMSO) were conducted in each series of experiments for concentrations in the range of $10^{-8}$ to $10 \mathrm{mM}$ to obtain a standard calibration curve. Thirty micro liters of acetylcholine iodide $(7.5 \mathrm{mM})$ was added to each well to initiate the reduction reaction and the development of the yellow color associated with the formation of the anionic species was recorded over time. The reaction was terminated by adding $10 \mu \mathrm{L}$ of a $4 \%(\mathrm{w} / \mathrm{v})$ solution of sodium dodecyl sulfate in $\mathrm{H}_{2} \mathrm{O}$.

Optical density (OD) of the supernatant was measured by use of a micro plate reader (Biotech, Winooski, VT 05404, USA) at $412 \mathrm{~nm}$. The AChE inhibitory activity was defined as follows:

$U=\frac{A_{B}-A_{S}}{A_{B}} \times 100 \%$

where $U$ is the percentage of AChE inhibitory activity, $A_{B}$ is the OD of the supernatant from the enzymatic reaction of the blank at $412 \mathrm{~nm}$, and $A_{S}$ is the OD of the supernatant from the enzymatic reaction of the sample at $412 \mathrm{~nm}$.

\subsection{Measurement of enantiospecific impact on the active site of AChE}

Fluorescent properties of AChE were measured by use of an F-7000 fluorescence spectrophotometer (Hitachi, Tokyo, Japan). One hundred micro liters of AChE was added to 900 micro liters of sodium phosphate buffer $(0.1 \mathrm{~mol} / \mathrm{L})$, and the resulting mixture was incubated on a thermo shaker at $37{ }^{\circ} \mathrm{C}$ for $30 \mathrm{~min}$. DMSO solutions of the samples $(1 \mathrm{mmol} / \mathrm{L})$ were added to this mixture, and 3D scan procedures for fluorescent intensity were monitored with an excitation wavelengths of $200-320 \mathrm{~nm}$ and emission wavelengths of $250-400 \mathrm{~nm}$. Scan speed were set at $30,000 \mathrm{~nm} / \mathrm{min}$ and PMT voltage was $550 \mathrm{~V}$. Slit widths for both wavelengths were set at $5 \mathrm{~nm}$. Assays were performed at $30 \mathrm{~s}$ intervals throughout the $3 \mathrm{D}$ scan process with 30 replicates.

The Stern-Volmer equation was used to investigate the fluorescence quenching in terms of quenching dynamics (Sklar et al., 1977). According to this equation, $F$ and $F_{O}$ represented the fluorescent intensities in the absence and presence of quenchable reagent, respectively. $K_{q}$ was a quenching speed constant and $C_{q}$ was the concentration of quenchable reagent. The parameter $\tau_{0}$ was calculated as the average fluorescence lifetime, but it was usually set at $10^{-8} \mathrm{~s}$ for biological macromolecules.

$F_{0} / F=1+K_{\mathrm{q}} \tau_{0} \mathrm{C}_{\mathrm{q}}$

Binding distances between fluorescent chemicals and quenchable reagents were also calculated by use of the Förster energy transfer equation, as follows:

$E=1-F / F_{0}=R_{0}^{6} /\left(R_{0}^{6}+\mathrm{r}_{0}^{6}\right)$

where $E$ represented the energy transfer efficiency, $R_{O}$ was the critical distance when $E$ was equal to $50 \%$, and $r_{O}$ is the actual binding distance.

\subsection{Statistical analysis}

Dose-response curve was fitted by use of a Sigmoidal Boltzmann model (see equation below) to obtain the effective concentration (EC) values (Rehmann et al., 1999),

$$
\mathrm{EC}=A_{2}+\frac{A_{1}-A_{2}}{1+\exp \left(\frac{x-x_{0}}{d_{x}}\right)}
$$

where $A_{1}$ was the initial value, $A_{2}$ was the final value, $x_{0}$ was the center value, and $d_{x}$ was the time constant (Table S1).

\section{Results and discussion}

\subsection{Binding mode of chiral OPs to human AChE}

Six pairs of OP enantiomers were successfully docked into the active site of human AChE by use of the Surflex-Dock programs. As shown in Table 1 , the binding affinities of all these compounds were predicted. The $S / R$-activities of the six OPs revealed that they all exhibited enantiospecific inhibition towards human AChE. The docking scores revealed that $R$-trichloronate, $S$-leptophos, $R$-fonofos, $S$-cycloheptyl methyl $S$-ethyl phosphonyl, $S$-isopropyl thiocholine and $S$-cycloheptyl thiocholine had higher binding affinities than the corresponding enantiomers. The simulated complexes suggested that Tryptophan86, Glycine120, Glycine121, Serine125, Glutamic acid202, Serine203, Tyrosine337 and Histidine447 were the key amino acid residues to form meaningful interactions with the 12 OPs (Fig. 1). Serine125 and Tyrosine337 were key H-bonding sites for the stabilization of ligands embedded in the binding site of AChE. Volume of active pocket was also considered in terms of the lipophilic potential surface of the active pocket, in which six pairs of enantiomers possessed different postures in the active site. Conformations of the bioactive conformers of different OPs revealed that their chiral center was closer to the catalytic triad (i.e., Glutamic acid202, Serine203, and Histidine447) than it was in the corresponding inactive conformations. The modeling results also revealed that the formation of an H-bonding interaction between OPs and Serine125 or Tyrosine337 enhanced the binding affinity of these compounds to human AChE. A comparison of the binding interactions of trichloronate (Fig. 1a) with those of leptophos (Fig. 1b) revealed that they both formed H-bond interactions to Serine125, but the hydroxyl group of the two bioactive enantiomers were closer to the oxyanion hole formed by Glycine120, Glycine121 and Serine125 than that of inactive enantiomers. It is noteworthy that a benzene ring bearing chlorine substituent would not be able to fit into the bottom of the active site due to the steric exclusion effect. There were common features among six bioactive conformations. The benzene ring in fonofos and the alicyclic ring in S-ethyl were parallel to the plane of the hydrophobic tryptophan 86 residue, suggesting that $R$-fonofos and $S$ cycloheptyl methyl $S$-ethyl phosphonyl were the major conformations (Fig. 1c and d). The bioactive conformations shown in Fig. 1e and $\mathrm{f}$ revealed that the chiral phosphorus atoms of these OPs were located in a narrow valley and that the chlorine leaving group pointed away from the entrance to the binding site. It is noteworthy that the AChE enzyme achieves enantiospecific recognition through a combination of specific hydrogen bonding interactions and complementary hydrophobic interactions with the ligand. Different binding modes of the two enantiomers could therefore be used to predict the inhibitory activity of new OPs towards AChE. The calculated non-bonding binding energies of these compounds could be used to explain the structure activity relationships of chiral OPs to interact with human AChE.

\subsection{A novel enantiomer-based virtual screening to discover AChE inhibitors}

A credible pharmacophore model was then established to investigate the toxic mechanisms of chiral OPs responsible for the enantioselectivity exhibited by AChE. The chemical feature-based pharmacophore model based on six bioactive enantiomers contained two hydrogen bond donors, a hydrogen bond acceptor and a hydrophobic feature 


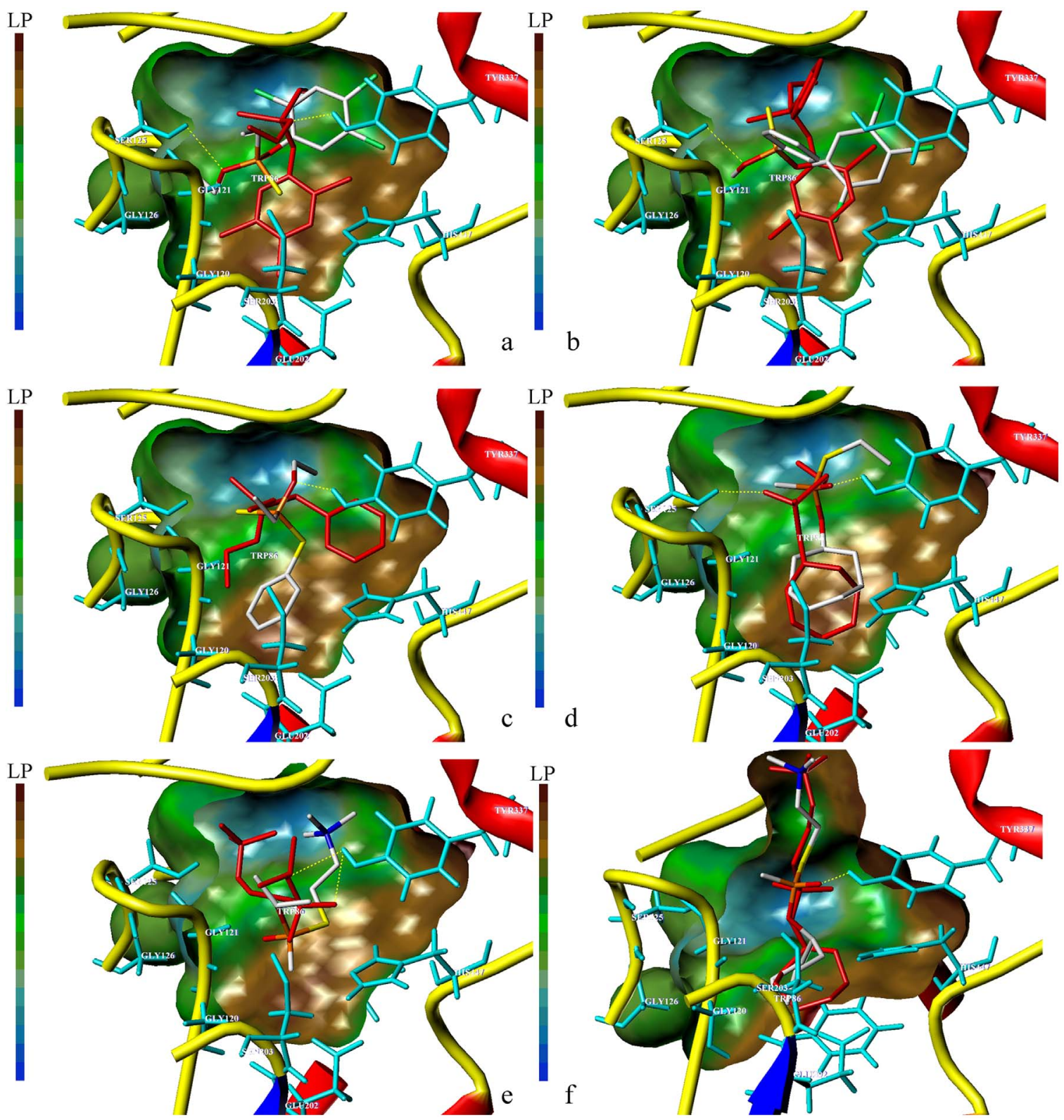

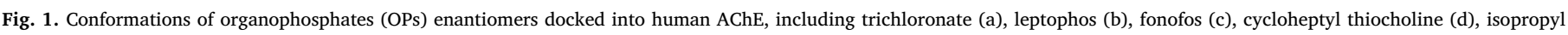

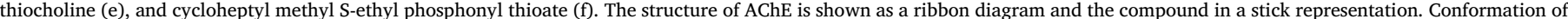

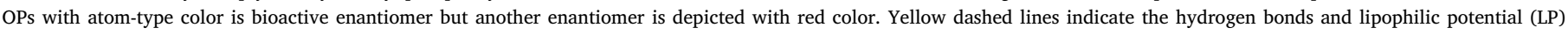
surfaces of active pocket are characterized with color ramps. The key amino acid residues colored cyan.

(Fig. 2). The total score of this model was 0.31 with a tolerance of $1.25 \AA$. Meanwhile, other six non-bioactive enantiomers for pharmacophore were not within a tolerance of $1.25 \AA$, which was demonstrated as a meaningful virtual screening. A four-feature pharmacophore model developed in a previous study composed of hydrophobic regions and $\mathrm{H}$ bond donor/acceptor and provided valuable insight for designing selective inhibitors for the treatment of Alzheimer's disease (Valasani et al., 2013). Barron et al. developed a structure-based pharmacophore model and generated 3D-fingerprint to describe 3D protein-ligand interactions in the active site gorge of AChE. The eight pharmacophore features in the binding site of AChE included 3 aromatic/hydrogen bond donor or hydrophobic features that interacted with Trp84 and Phe330, a hydrogen bond donor feature that interacted with carbonyl oxygen of His440, a hydrogen bond acceptor feature that interacted with Gly118, Gly119, and Ala201, and 3 hydrophobic features in acyl pocket (Phe288 and Phe290) (Lee and Barron, 2015). The type of those pharmacophore models also contained four location-specific features with stereospecificity (Mesecar and Koshland, 2000). It was concluded that the tetrahedral structure was a basis to determine the enantiospecificity of target enzyme. It seemed plausible that the primary 3D pharmacophore model could provide a good platform for further database searching and the rational design of new AChE inhibitors.

The four-location model developed in the current study was used to conduct a searching query against the National Cancer Institute (NCI) database for anti-HIV and anti-cancer assay data. One of the main advantages of virtual screening is that it allows for the rapid and lowcost screening of large numbers of compounds in silico, making it a useful tool for the prescreening of potential lead compounds. Tolerance value of the pharmacophore model was modified from 0.75 to $0.25 \AA$ in this particular case to enhance its accuracy. Twenty-four compounds were found, including 1-naphthylacetic anhydride (M10202), 2-(dimethylamino)-2-oxo-1-phenylethyl(phenyl)phosphinic acid (M130076), ethyl bis(2,2-dimethyl-1-aziridinyl)phosphorylcarbamate (M51325), dimethyl bicyclo[2.2.1]hept-2-ylphosphonate (M 138990), methyl 


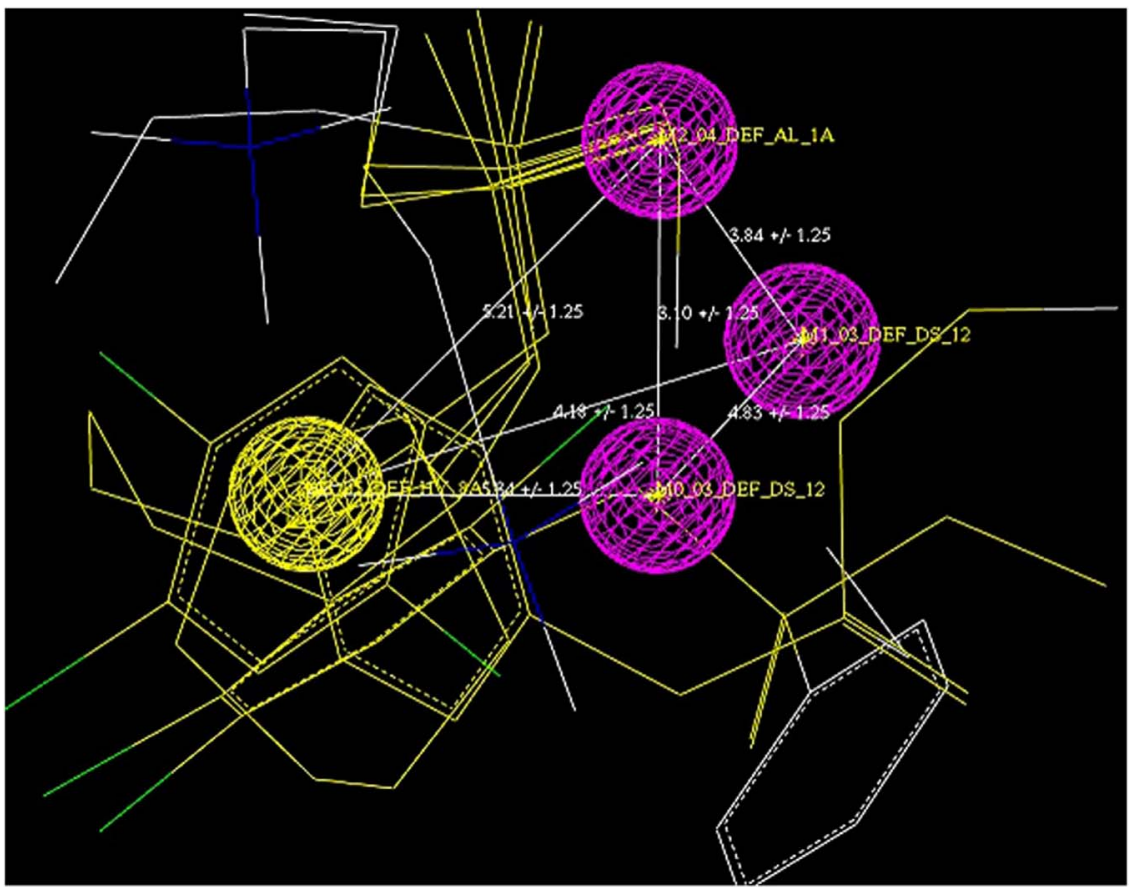

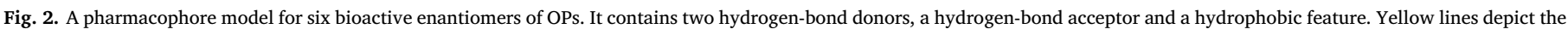
maximum common substructures.

(diphenylphosphorothioyl)(phenyl)acetate (M130079), O-ethyl O-(4(hydroxy(oxido)amino)phenyl) phenylphosphonothioate (M404840), 4-methylbenzenesulfinic acid (4871), ethyl 2-((phenylsulfonyl) methyl)acrylate (M623994), 1-tert-butyl-4-((chlorooxy)sulfinyl)benzene (M8544), No name (M122496), N-(cyclobutyl(1-pyrrolidinyl) methylene)-8-quinolinesulfonamide (M116974), N'-benzoyl-N'-methylbenzenesulfonohydrazide (M37428), 1-(((bis(2-chloroethyl)amino)oxy) sulfinyl)-4-methylbenzene (M23809), 7-((phenylsulfonyl)amino)bicyclo[2.2.1]hept-2-yl acetate (M98587), N-(4-aminophenyl)-N,4-dimethylbenzenesulfonamide (M105638), N,4-dimethyl-N-phenylbenzenesulfonamide (M2225), 4-aminophenylphosphonous acid (M3625), 2(1,2-dihydro-5-acenaphthylenyl)acetamide (M17466), 1-(4(methylthio)phenyl)-2-(1-quinolin-1-yl)ethanone (M35029), (2-hydroxy-3-methoxy-5-methylphenyl)(4-phenyl-4,5-dihydro-1H-pyrazol-3-yl) methanone (M404854), 2-(1,2,2-triisopropoxyethoxy)propane (M21542), allyl 3-phenyl-2-oxiranecarboxylate (M68483), diethyl 2,2dibenzylmalonate (M8726), and dimethyl 8-oxo-2-cyclooctene-1,2-dicarboxylate (M119539). Most of those compounds contained chiral or pro-chiral atoms. Three structurally diverse compounds from this small set were purchased to measure their inhibitory activity towards AChE. We would have preferred to purchase all of these compounds to measure their inhibitory activity towards AChE, but some of these compounds were not commercially available.

\subsection{Measurement of AChE activity by competitive inhibitory assay and} fluorescence assay

Potency of OPs to inhibit activity of AChE was measured and compared to theoretical results. Toxicities of these three compounds were evaluated by use of an AChE competitive inhibitory assay, which allowed us to generate dose response curves of them. They were EPN, 1naphthaleneacetic anhydride and $N$, 4-dimethyl- $N$-phenyl-benzenesulfonamide. Tacrine hydrochloride was also tested as positive control. This method has been used routinely for many years to monitor terrestrial and freshwater aquatic systems as an indicator for the bioactivity of OPs (Fulton and Key, 2001). As shown in Fig. 3, the logarithm of median effective concentration of tacrine (positive control) was determined as -6.48. 1-naphthaleneacetic anhydride, EPN and
$N$, 4-dimethyl- $N$-phenyl-benzenesulfonamide exhibited moderate inhibitory activity towards AChE compared with tacrine, with $\log \mathrm{EC}_{50}$ values of $-2.91,-2.46$ and -5.22 , respectively. The activity of 1 naphthaleneacetic anhydride was attributed to its hydrophobic interaction with AChE, whereas the pro-chiral $N$, 4-dimethyl- $N$-phenylbenzenesulfonamide compound has higher activities. Meanwhile, our measurement for EPN is consistent with reports from the literature concerning the toxicity of this compound towards mammals (Devillers, 2004). However, the different enantiomers of this compound have been reported to show different toxicities (Johnson and Read, 1987; Tsugawa et al., 2000). Further study is therefore required to assess the environmental risks associated with the different enantiomers of EPN.

A fluorescence assay on human AChE was performed by use of an F7000 fluorescence spectrophotometer (Hitachi, Tokyo, Japan). As shown in Fig. 4a, fluorescence spectrum of AChE contained an emission peak at $320 \mathrm{~nm}$ with an excitation wavelength of $230 \mathrm{~nm}$, which is a typical characteristic of the tryptophan. However, the result of a 3D fluorescence lifetime scan in the presence of EPN with a fixed excitation wavelength of $270 \mathrm{~nm}$ and a reaction time of $900 \mathrm{~s}$ revealed that the fluorescence intensity of tryptophan decreased from $333.8 \mathrm{~nm}$ to $128.6 \mathrm{~nm}$ (Fig. 4b). This finding indicated that fluorescence had been quenched during the reaction time. The $K q$ value of this system was determined to be $1.61 \times 10^{12} \mathrm{~L} / \mathrm{mol} \mathrm{s}$ in Eq. (2), which was much greater than the maximum value allowed for a macromolecule of $2.0 \times 10^{10} \mathrm{~L} / \mathrm{mol} \mathrm{s}$. Fluorescence quenching of AChE was therefore attributed to the formation of an EPN-AChE complex, that was involved in static quenching. The binding distance $r_{O}$ was determined to be 0.924 $R_{O}\left(0.5 R_{O}<r_{O}<1.5 R_{O}\right)$, which gave an energy-transfer efficiency $E$ of $61.6 \%$ in Eq. (3). It suggested that EPN was an effective quenchable reagent for AChE, because it might bind to its hydrophobic cavity. Compared with the fluorescence quenching of Tyrosine, fluorescence quenching of tryptophan was greater, with a considerable Einstein shift occurring during the quenching process. This phenomenon shows that the tryptophan plays an important role in the binding of EPN to AChE, and that the interaction between these two groups is weaker than that expected based on the Einstein shift.

Given that an isomer of EPN could lead to a greater decrease in the polarity of tryptophan, it should be possible to confirm the differences 


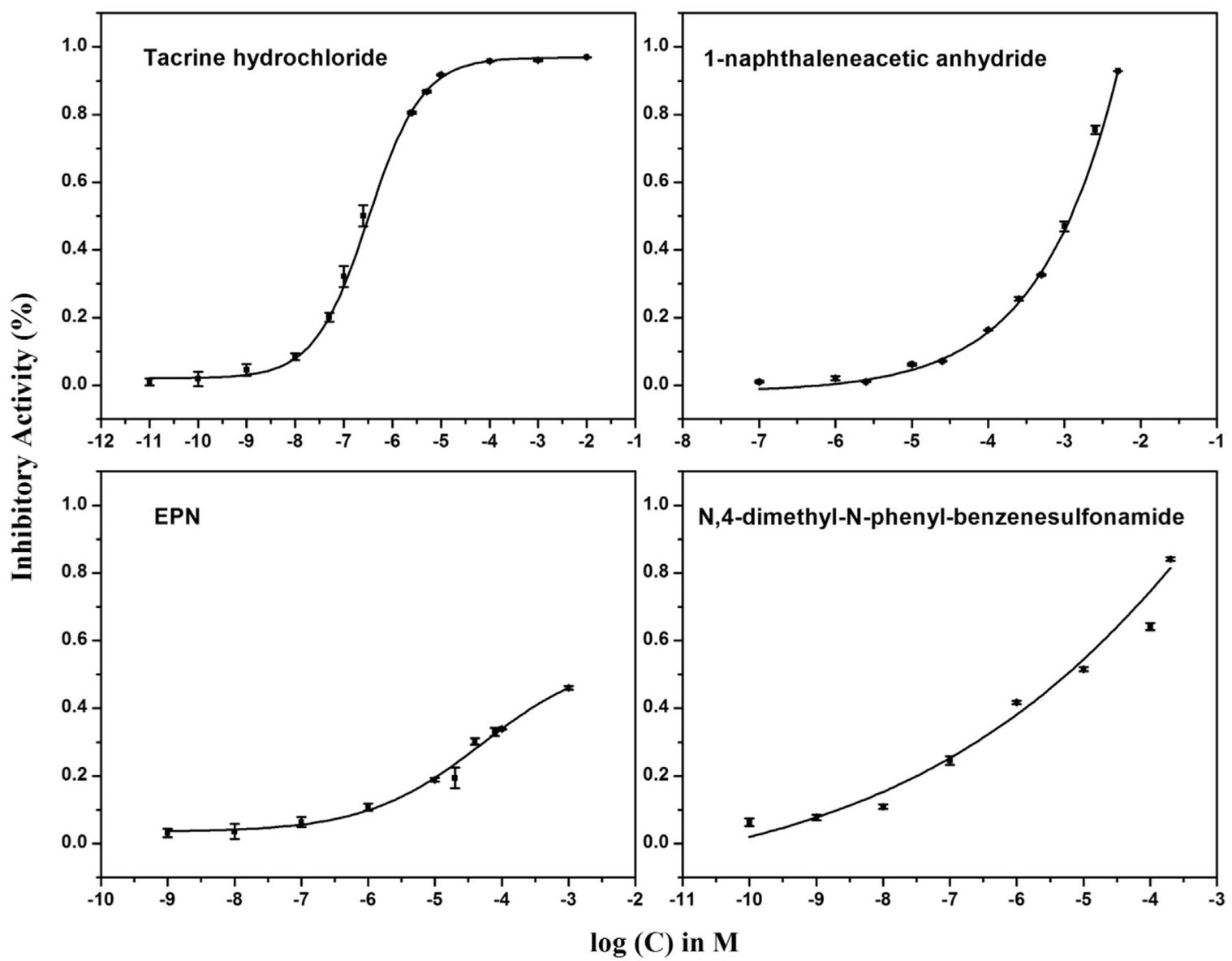

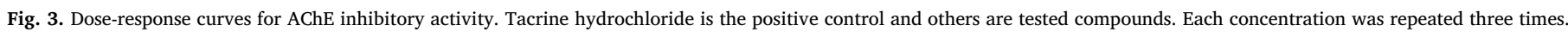
Error bars indicate standard error. Modeled data for the compounds are shown as a solid line.

in the binding of enantiomers without chiral separation. Binding modes of $S$ - and $R$-EPN were predicted by use of a flexible docking method. As shown in Fig. 5, $S$-EPN was buried deep inside the hydrophobic cavity of human AChE, with a water molecule acting as a hydrogen bond bridge between $S$-EPN and Serine125. The benzene ring of $S$-EPN was located in close proximity to the hydrophobic environment of Tryptophan 86 and it could form credible $\pi-\pi$ interactions with the bottom of the pocket. In contrast, $R$-EPN adopted the opposite conformation to $S$-EPN, with its nitrobenzene substituent occupying the catalytic site, making it difficult to form a catalytic intermediate through a covalent process. These findings therefore confirmed that $S$ -
EPN exhibited greater inhibitory activity towards AChE than $R$-EPN.

\section{Conclusions}

Molecular simulation, on basis of stereochemistry of enzyme, provides a sensitive process to identify the bioactive enantiomers of OPs to AChE. A virtual screening method was then developed to search new inhibitors to human AChE, including EPN, 1-naphthaleneacetic anhydride and $N$, 4-dimethyl- $N$-phenyl-benzenesulfonamide. Some in vitro assays were performed to determine the inhibitory activity as well as the binding modes of different enantiomers. This study gives a novel

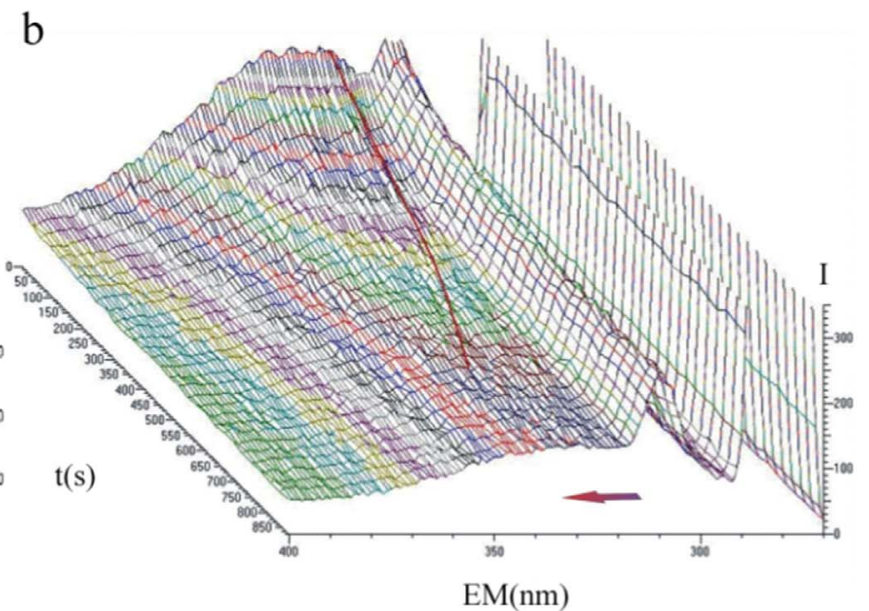

b

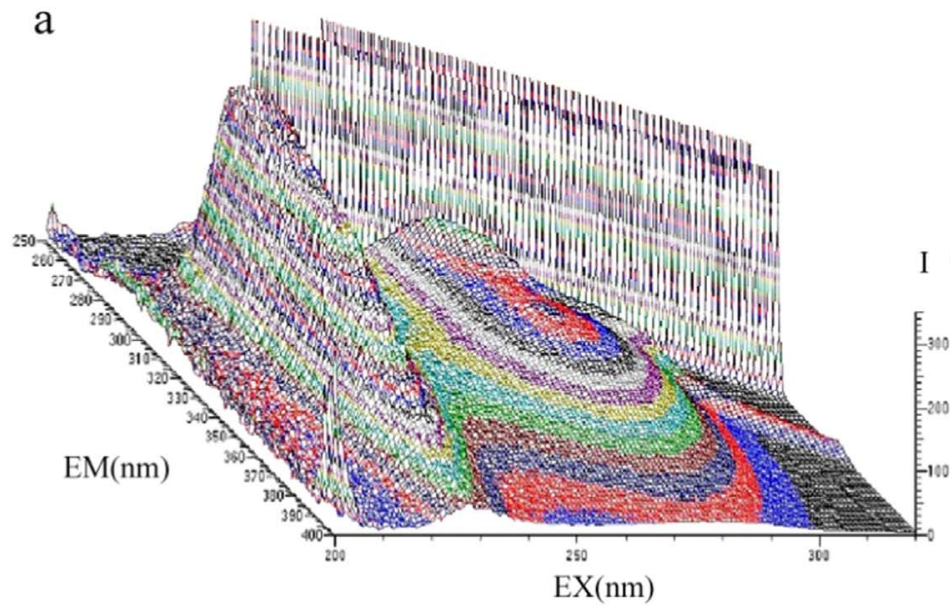

Fig. 4. 3-D fluorescence scan contour (a) and 3-D time scan contour (b) for fluorescence quenching of AChE in the presence of ethyl p-nitrophenyl phenylphosphonate (EPN). 

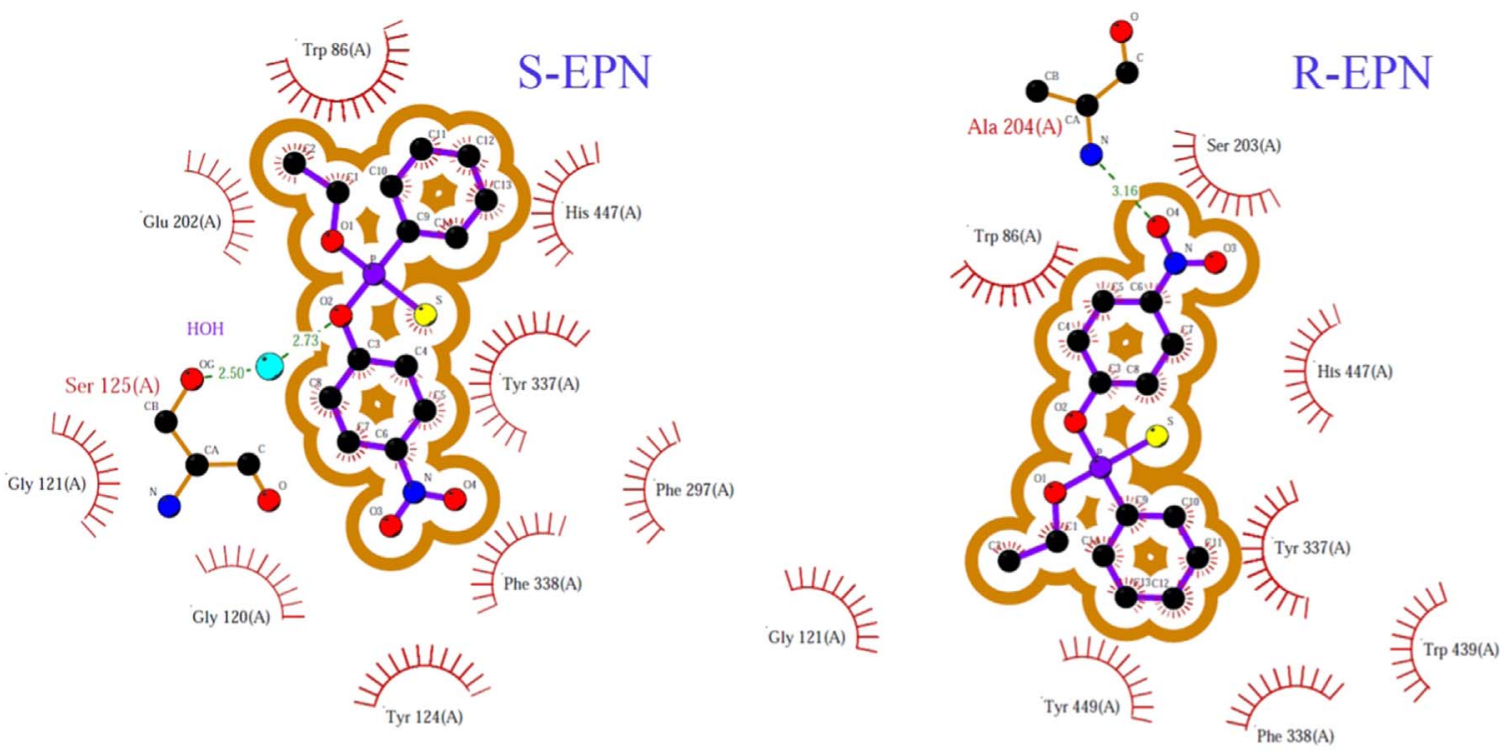

Key

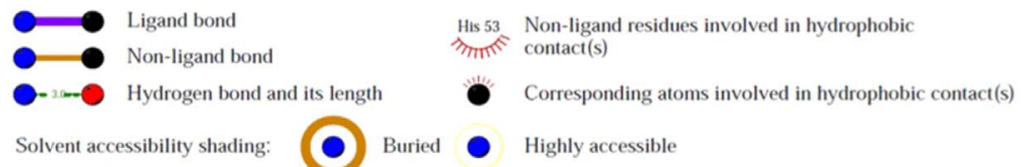

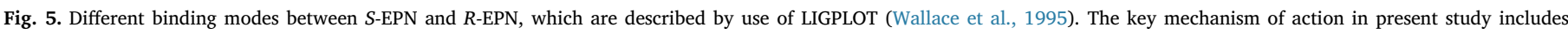
hydrogen bond and its length, hydrophobic contacts and solvent accessibility.

\section{direction to evaluate the stereo selective toxicity of chiral compounds to} their target proteins.

\section{Acknowledgements}

This work was supported in part by the National Natural Science Foundation (41673087, 21507120, and 21277164) and Chinese Academy of Sciences (KZCX2-YW-BR-25 and YSW2013B01).

\section{Appendix A. Supporting information}

Supplementary data associated with this article can be found in the online version at doi:10.1016/j.ecoenv.2016.12.035.

\section{References}

Boter, H.L., Dijk, C. v., 1969. Stereospecificity of hydrolytic enzymes on reaction with asymmetric organophosphorus compounds. 3. The inhibition of acetylcholinesterase and butyrylcholinesterase by enantiomeric forms of sarin. Biochem. Pharmacol. 18, 2403-2407.

Brown, G.G., et al., 1989. In vivo 31P NMR profiles of Alzheimer's disease and multiple subcortical infarct dementia. Neurology 39, 1423-1427.

Carletti, E., et al., 2010. Structural evidence that human acetylcholinesterase inhibited by tabun ages through O-dealkylation. J. Med. Chem. 53, 4002-4008.

Cavalli, A., et al., 2004. A computational study of the binding of propidium to the peripheral anionic site of human acetylcholinesterase. J. Med. Chem. 47, 3991-3999.

de Castro, A.A., et al., 2016. Molecular docking, metal substitution and hydrolysis reaction of chiral substrates of phosphotriesterase. Comb. Chem. High Throughput Screen. 19, 334-344.

Devillers, J., 2004. Prediction of mammalian toxicity of organophosphorus pesticides from QSTR modeling. SAR QSAR Environ. Res. 15, 501-510.

Dvir, H., et al., 2002. 3D structure of Torpedo californica acetylcholinesterase complexed with huprine $\mathrm{X}$ at 2.1 A resolution: kinetic and molecular dynamic correlates. Biochemistry 41, 2970-2981.

Ellman, G.L., et al., 1961. A new and rapid colorimetric determination of acetylcholinesterase activity. Biochem. Pharmacol. 7, 88-95.

Fulton, M.H., Key, P.B., 2001. Acetylcholinesterase inhibition in estuarine fish and invertebrates as an indicator of organophosphorus insecticide exposure and effects. Environ. Toxicol. Chem. 20, 37-45.

Guo, J.X., et al., 2006. Mechanistic insight into acetylcholinesterase inhibition and acute toxicity of organophosphorus compounds: a molecular modeling study. Chem. Res.
Toxicol. 19, 209-216.

Harel, M., et al., 1993. Quaternary ligand binding to aromatic residues in the active-site gorge of acetylcholinesterase. Proc. Natl. Acad. Sci. USA 90, 9031-9035.

Hosea, N.A., et al., 1995. Specificity and orientation of trigonal carboxyl esters and tetrahedral alkylphosphonyl esters in cholinesterases. Biochemistry 34, 11528-11536.

Jain, A.N., 2003. Surflex: Fully automatic flexible molecular docking using a molecular similarity-based search engine. J. Med. Chem. 46, 499-511.

Johnson, M.K., Read, D.J., 1987. The influence of chirality on the delayed neuropathic potential of some organophosphorus esters: neuropathic and prophylactic effects of stereoisomeric esters of ethyl phenylphosphonic acid (EPN oxon and EPN) correlate with quantities of aged and unaged neuropathy target esterase in vivo. Toxicol. Appl. Pharmacol. 90, 103-115.

Kryger, G., et al., 1998. Three-dimensional structure of a complex of E2020 with acetylcholinesterase from Torpedo californica. J. Physiol. 92, 191-194.

Lee, S., Barron, M.G., 2015. Development of 3D-QSAR model for acetylcholinesterase inhibitors using a combination of. Toxicol. Sci. 148, 60-70.

Lee, S., Barron, M.G., 2016. A mechanism-based 3D-QSAR approach for classification and prediction of acetylcholinesterase inhibitory potency of organophosphate and carbamate analogs. J. Comput. Aided Mol. Des. 30, 347-363.

Li, J., et al., 2014. Water-driven micromotors for rapid photocatalytic degradation of biological and chemical warfare agents. ACS Nano 8, 11118-11125.

Liu, W., et al., 2005. Enantioselectivity in environmental safety of current chiral insecticides. Proc. Natl. Acad. Sci. USA 102, 701-706.

Liu, W., et al., 2006. Separation and aquatic toxicity of enantiomers of the organophosphorus insecticide trichloronate. Chirality 18, 713-716.

Mesecar, A.D., Koshland, D.E., 2000. Structural biology: a new model for protein stereospecificity. Nature 403, 614-615.

Millard, C.B., et al., 1999a. Reaction products of acetylcholinesterase and VX reveal a mobile histidine in the catalytic triad. J. Am. Chem. Soc. 121, 9883-9884.

Millard, C.B., et al., 1999b. Crystal structures of aged phosphonylated acetylcholinesterase: nerve agent reaction products at the atomic level. Biochemistry 38, 7032-7039.

Nillos, M.G., et al., 2007. Enantioselective acetylcholinesterase inhibition of the organophosphorous insecticides profenofos, fonofos, and crotoxyphos. Environ. Toxicol. Chem. 26, 1949-1954.

Ordentlich, A., et al., 1998. Functional characteristics of the oxyanion hole in human acetylcholinesterase. J. Biol. Chem. 273, 19509-19517.

Ordentlich, A., et al., 1999. Exploring the active center of human acetylcholinesterase with stereomers of an organophosphorus inhibitor with two chiral centers. Biochemistry 38, 3055-3066.

Ordentlich, A., et al., 2005. The role of AChE active site gorge in determining stereoselectivity of charged and noncharged VX enantiomers. Chem. Biol. Interact. 157-158, 191-198.

Pilger, C., et al., 2001. Accurate prediction of the bound conformation of galanthamine in the active site of Torpedo californica acetylcholinesterase using molecular docking. J. 
Mol. Graph Model. 19 (288-96), 374-378.

Quinn, D.M., 1987. Acetylcholinesterase: enzyme structure, reaction dynamics, and virtual transition states. Chem. Rev. 87, 955-979.

Rehmann, K., et al., 1999. Applicability of a yeast oestrogen screen for the detection of oestrogen-like activities in environmental samples. Chemosphere 38, 3303-3312.

Rosenberry, T.L., et al., 1999. A steric blockade model for inhibition of

acetylcholinesterase by peripheral site ligands and substrate. Chem. Biol. Interact. 119-120, 85-97.

Singh, B.K., 2009. Organophosphorus-degrading bacteria: ecology and industrial applications. Nat. Rev. Microbiol. 7, 156-164.

Sklar, L.A., et al., 1977. Conjugated polyene fatty acids as fluorescent probes: binding to bovine serum albumin. Biochemistry 16, 5100-5108.

Sussman, J.L., et al., 1991. Atomic structure of acetylcholinesterase from Torpedo californica: a prototypic acetylcholine-binding protein. Science 253, 872-879.

Tenberken, O., et al., 2010. Chromatographic preparation and kinetic analysis of interactions between tabun enantiomers and acetylcholinesterase. Toxicol. Lett. 195, 142-146.

Tsai, P.C., et al., 2010. Stereoselective hydrolysis of organophosphate nerve agents by the bacterial phosphotriesterase. Biochemistry 49, 7978-7987.

Tsugawa, W., et al., 2000. Improvement of enantioselectivity of chiral organophosphate insecticide hydrolysis by bacterial phosphotriesterase. Appl. Biochem. Biotechnol. 84-86, 311-317.

Valasani, K.R., et al., 2013. Acetylcholinesterase inhibitors: structure based design, synthesis, pharmacophore modeling, and virtual screening. J. Chem. Inf. Model. 53, 2033-2046.

Wallace, A.C., et al., 1995. LIGPLOT: a program to generate schematic diagrams of protein-ligand interactions. Protein Eng. 8, 127-134.

Wang, C., et al., 2010. Enantioselective interaction with acetylcholinesterase of an organophosphate insecticide fenamiphos. Chirality 22, 612-617.

Wong, L., et al., 2000. Mechanism of oxime reactivation of acetylcholinesterase analyzed by chirality and mutagenesis. Biochemistry $39,5750-5757$.

Yen, J.H., et al., 2003. Separation and toxicity of enantiomers of organophosphorus insecticide leptophos. Ecotoxicol. Environ. Saf. 55, 236-242.

Zhang, A., et al., 2014. Enantioselective interaction of acid alpha-naphthyl acetate esterase with chiral organophosphorus insecticides. J. Agric. Food Chem. 62 1477-1481.

Zhang, Y., et al., 2002. Role of the catalytic triad and oxyanion hole in acetylcholinesterase catalysis: an ab initio QM/MM study. J. Am. Chem. Soc. 124, 10572-10577. 